\title{
Análisis de las valoraciones del alumnado para repensar la autoridad docente y la formación para la ciudadanía
}

Analysis of student assessment to rethink the
teaching authority and training for citizenship

\author{
Gladys Merma-Molina ${ }^{1}$ \\ gladys.merma@ua.es \\ Diego Gavilán Martín \\ diego.gavilan@ua.es \\ Universidad de Alicante, España
}

\section{Resumen:}

El incremento de la indisciplina y de la violencia escolar, la pérdida de autoridad del maestro y la falta de civilidad de los jóvenes es preocupante. Con el fin de analizar las causas y consecuencias de estos hechos, este estudio tienen como objetivos: 1) identificar los rasgos que caracterizan la autoridad de los profesores, 2) determinar los comportamientos que generan dichos rasgos en los alumnos y 3 ) analizar las relaciones entre la autoridad docente y ciudadanía. El instrumento de recogida de datos es el cuestionario Autoridad docente y convivencia, validado por el Grupo de investigación sobre indisciplina y violencia en la educación. La muestra estuvo constituida por 738 alumnos. Las dimensiones analizadas son: competencia docente, comportamiento ejemplar, personalidad y actitudes. Los hallazgos muestran que los estudiantes destacan como rasgos de la autoridad de sus docentes: 1) sus actitudes: sabe relacionarse con los estudiantes y no es excluyente, se intere-

\begin{abstract}
:
The increase in indiscipline and school violence, the loss of teacher authority and the lack of civility in young people is worrisome. In order to search and analyse the causes and consequences of these events, this study aims to: 1 ) identify the features that characterise teachers' authority according to students' opinion; 2) determine what type of behaviours these characteristics generate in students; and 3) analyse the possible relationships between teaching authority and citizenship. The data collection instrument is the Teaching Authority and Coexistence Questionnaire, designed and validated by the Research Group on Indiscipline and Violence in Education. The sample consisted of 738 students. The dimensions that are analysed are: teaching competence, exemplary behaviour, personality and attitudes. The findings reveal that students remark the following features regarding their teachers' authority: 1) their attitudes: they know how to relate to students, they participate
\end{abstract}

1 Dirección para correspondencia (correspondence address):

Gladys Merma Molina. Facultad de Educación. Universidad de Alicante. Carretera de San Vicente del Raspeig, s/n. 03690, Alicante (España). 
Análisis de las valoraciones del alumnado para repensar la autoridad docente y la formación para la ciudadanía

Gladys Merma-Molina y Diego Gavilán Martín

sa por cada alumno, busca la armonía y trato personal, 2) su dominio de la asignatura, el empleo de metodologías activas y su interés porque los alumnos aprendan; y 3) la personalidad del docente: es exigente, autoexigente e imparcial. Asimismo, sostienen que los maestros no tienen una conducta ejemplar, no son justos cuando sancionan, no respetan a los estudiantes y su conducta no es conforme aconsejan. De las correlaciones entre la autoridad docente y las actitudes de los alumnos, se concluye que las características del docente influyen en el comportamiento de los alumnos, en el clima del aula y en el rendimiento académico.

\section{Palabras clave:}

Autoridad docente; convivencia; ciudadanía; educación. in activities and they integrate all students, they are interested in all students, they seek harmony and treat each student on a personal level, 2) their mastery of the subject, the use of active methodologies and their interest in increasing students' knowledge, and 3) the personality of the teachers: they are demanding, self-demanding and impartial. Other aspects students identify include the facts that teachers do not have an exemplary behaviour, they are not fair with their punishments, they do not respect all students and they do not behave as they ask students to behave. The correlations between teaching authority and the attitudes of the students reveal that the characteristics of the teacher influence students' behaviour, classroom climate and academic performance.

\section{Keywords:}

Teaching authority; coexistence; citizenship; education.

\section{Résumé:}

L'augmentation de l'indiscipline et de la violence à l'école, la perte d'autorité de l'enseignant et le manque de civisme des jeunes sont préoccupants. Afin de rechercher et d'analyser les causes et les conséquences de ces événements, cette étude vise à: 1) identifier les traits qui caractérisent l'autorité des enseignants, selon l'opinion des étudiants, 2) déterminer le type de comportement que ces caractéristiques génèrent chez les étudiants et 3) analyser les relations possibles entre l'autorité enseignante et la citoyenneté. L'instrument de collecte de données est le questionnaire sur l'autorité d'enseignement et la coexistence, conçu et validé par le groupe de recherche sur l'indiscipline et la violence dans l'éducation. L'échantillon était composé de 738 étudiants. Les dimensions analysées sont les suivantes: compétence pédagogique, comportement exemplaire, personnalité et attitudes. Les résultats les plus importants montrent que les élèves soulignent, en tant que caractéristiques de l'autorité de leurs enseignants: 1) leurs attitudes: ils savent comment établir des relations avec les élèves sans être exclusifs, s'intéressent à chaque élève et recherchent l'harmonie et la considération personnelle de l'étudiant; 2) leur mâ̂trise du sujet, l'utilisation de méthodologies actives et leur intérêt pour que les étudiants apprennent; et 3) la personnalité de l'enseignant: il est exigeant, autonome et impartial. Par contre, les élèves soutiennent que les enseignants n'ont pas un comportement exemplaire, ne punissent pas les fautes avec justice, ne respectent pas tous les élèves et que leur comportement n'est pas conforme aux conseils qu'ils donnent. Les corrélations existant entre l'autorité enseignante et les attitudes des élèves permettent de conclure que les caractéristiques de l'enseignant influent sur le comportement des élèves, sur le climat de la salle de classe et sur les résultats scolaires.

\section{Mots-clés:}

Autorité d'enseignement; coexistence la citoyenneté; éducation.

Fecha de recepción: 1-6-2018

Fecha de aceptación: 15-9-2018 


\section{Introducción}

Diferentes estudios realizados en España sobre la convivencia escolar en la Educación Primaria y en la Educación Secundaria muestran que los profesores se quejan de la conducta negativa de sus alumnos, del desinterés académico, de los comportamientos disruptivos, de la falta de respeto, de la desobediencia y de una actitud contraria a las normas del centro y del aula (Calvo, 2011; Díaz-Aguado, 2013; Díaz-Aguado, Martínez, y Martín, 2010; Urbina, Simón, y Echeita, 2011; Simón, Gómez, y Alonso, 2013). Álvarez, Castro, González-González, Álvarez y Campo (2016) sostienen que los profesores tienen serias dificultades para impartir las clases y que esta problemática se ha incrementado drásticamente en los últimos años.

La situación es similar en otros países europeos (Maguire, Ball, y Braun, 2010; McGarr, O'Grady, y Guifolyle, 2016); así, Maphosa y Mammen (2011) afirman que en el Reino Unido los alumnos, en general, son ruidosos e irrespetuosos con los profesores. El uso del lenguaje grosero e incluso el acoso, la violencia física y los delitos contra la propiedad privada, perpetrados por jóvenes en edad escolar también se confirman en los Estados Unidos (McMahon et al., 2014).

EI 65\% de los profesores, en España, admite tener dificultades para desarrollar las clases debido a que no pueden controlar los comportamientos de los estudiantes. A estas conductas de desobediencia de las normas del centro/aula se ha denominado indisciplina y, según la opinión de los docentes, es uno de los problemas más álgidos en la educación.

Además, en la sociedad actual hay una visión extendida muy crítica a propósito de la falta de civilidad de los adolescentes caracterizada por su escaso interés y/o ignorancia respecto a los asuntos de la colectividad, su egoísmo generacional, su falta de respeto por las personas mayores y por sus padres, y su incapacidad para respetar las normas y la autoridad dentro y fuera del aula (Ivanenko, 2017; Martuccelli, 2016). Los expertos señalan que esto podría estar vinculado con una educación para la ciudadanía y la convivencia deficientes y con la pérdida de la autoridad docente que se ve reforzada por los medios de comunicación (Palomares, 2008; Ruiz, 2013).

Con relación al maestro, este está cuestionado por la sociedad, por los medios de comunicación, por los políticos, padres y hasta 
por los alumnos. Los docentes reconocen que han perdido autoridad, lo que frecuentemente se asocia con el incremento de las problemáticas antes mencionadas (la disrupción, la indisciplina y la violencia escolar) (Gallego, Acosta, Villalobos, López, y Giraldo, 2016; Torrubia, Guzón, y Alfonso, 2016). Esta situación es nueva y ha creado tensiones en la escuela que se evidencian en las dificultades que experimentan los docentes para ser reconocidos como una autoridad pedagógica.

En la escuela tradicional los profesores gozaban de una legitimidad ante los estudiantes y ante las familias; los niños abandonaban su mundo particular y accedían a la cultura escolar que interiorizaban sin resistencias. Sin embargo, en la escuela de hoy los estudiantes presentan una poliarquía de valores (Dubet, 2006, 2007) que exige que el profesorado les proporcione un espacio para reconocer su individualidad; de esta manera la autoridad del profesor ya no sirve para todo el grupo sino que debe ser reconocida y aceptada por cada estudiante (Zamora-Poblete, Mesa-Pardo, y Cox-Vial, 2015). A esto se añade que existen una serie de dificultades en la formación del maestro y, concretamente, en el desarrollo del proceso de ciudadanización en su formación inicial dentro de las cuales destaca la presentación de experiencias irrelevantes para el estudiantado como expresión del ejercicio de la ciudadanía (LadsonBillings, 2004).

El no abordaje o el escaso tratamiento de temas polémicos como los nuevos modelos familiares, la orientación sexual, la discriminación racial y cultural en la escuela, la escasa atención a temas de carácter global, y la falta de entrenamiento en el desarrollo de un pensamiento crítico revela el predominio de un enfoque de aprendizaje pasivo. Estas dificultades se relacionan con la conformación de un concepto de ciudadanía bastante restringido, visible incluso en profesores y estudiantes de universidades, quienes la circunscriben a dimensiones tales como votar en las elecciones y obedecer las leyes. En suma, el enfoque de las competencias ciudadanas en la formación inicial no se basa en una concepción constructivista del aprendizaje y no se desarrollan habilidades a partir de la realidad y de la práctica educativa. 


\section{Marco teórico}

\section{La autoridad docente para la construcción de la ciudadanía}

La convivencia es una manera de actuar que privilegia la hospitalidad y la civilidad; aunque la ciudadanía no se reduce a la hospitalidad, la requiere; no obstante, exige la civilidad. La escuela es el espacio social privilegiado para fomentar la hospitalidad y la civilidad, para desarrollar el comportamiento moral, y para comenzar a practicar y a construir la ciudadanía inclusiva.

La ciudadanía inclusiva consiste en promover la convivencia basada en el respeto a las diferencias, con la participación democrática de todos los miembros del centro escolar. La convivencia con el otro empieza por su

Reconocimiento como igual a uno mismo, que es sobre todo moral: creemos al otro igual a nosotros cuando le atribuimos la misma capacidad de pensar y sentir, de disfrutar y de sufrir, de desear y de dar; y lo sentimos miembro de nuestra misma comunidad cuando creemos que sus normas, formas de vida y valores son respetables y compatibles con los nuestros (Fernández, 2008, p. 6).

La escuela es una sociedad en pequeño constituida por personas que tienen y ejercen autoridad (autoridad administrativa, autoridad pedagógica y autoridad directiva) y por niños y jóvenes que provienen de diversas culturas. En esta institución, que se caracteriza por la diversidad de orígenes de sus miembros y por la multiplicidad de funciones, los valores de la democracia se desarrollan en función del modo en que proyecta la autoridad. Allí se construyen los principios de igualdad, tolerancia y respeto a las diferencias en las relaciones entre sus miembros; se forman ciudadanos que conozcan sus derechos, que aprendan a respetar los derechos de los otros y que participen en la construcción del bien común. En consecuencia, la escuela educa al ciudadano del mañana, pero también incita al alumno a experimentar y ejercer en la escuela de hoy una vida responsable y comprometida con la colectividad, ya que el hecho de ser capaz de convivir con el otro y de aprender la urbanidad implica el ejercicio de la ciudadanía en su forma elemental (Tassin, 2011).

El término autoridad no es fácil de definir. Muchas veces se utiliza 
como sinónimo de poder y otras como dominación; también se emplea para referirse a las relaciones centradas en el respeto y la admiración hacia otros, así como en aquellas situaciones impregnadas de rasgos arbitrarios.

Pese a su importancia, la autoridad y sus repercusiones en el éxito o fracaso del hecho educativo han sido poco estudiados. El término etimológicamente proviene del Latín, augere, que significa "acrecentar, promover, aumentar, hacer progresar", pero aún se sigue confundiendo con coacción e imposición, con obediencia, miedo y castigo (Tahull, y Montero, 2013).

Díaz (1998) explica con acierto y buen criterio que la autoridad es el "el bienquerer" y sostiene que quien sabe entregar su cariño se convierte en autoridad y que solo es deseable la autoridad que auxilia, la que aúpa, lo que no impedirá que ella sepa decir en un momento determinado una palabra dura, pero sin aspavientos ni histerias, con buenas maneras, aunque con firmeza. En el proceso de enseñanza-aprendizaje es necesaria la autoridad que no es autoritaria, sino aquella que es sustantiva e inherente.

Las escasas investigaciones que han indagado la autoridad del docente se han centrado en abordar las diferencias entre autoridad y autoritarismo (Barba, 2009). Otras se han referido a la forma en que los profesores conciben la autoridad y cómo la construyen (Harjunen, 2009; Zamora y Zerón, 2010), pero son pocas las investigaciones que hayan examinado cómo perciben los alumnos la autoridad del maestro y en qué medida los rasgos identificados influyen o no en el comportamiento de los estudiantes.

En este estudio, entendemos que la autoridad del profesor se basa en el reconocimiento que le otorgan los estudiantes al aceptar sus demandas (Arend, 1996; Kojève, 2006; Pierella, 2015) y que educar no es imponer, sino es convencer y ayudar a crecer; por tanto, la autoridad no implica principios en sí mismos sino atributos, cualidades y rasgos reconocidos. Espot (2006) ya defendía que la educación tiene que usar necesariamente la auctoritas y la potestas, que son dos elementos necesarios para gestionar el aula.

Tradicionalmente la acción educativa surgía del ejercicio de una autoridad docente institucionalmente establecida que no requería del consenso estudiantil, lo que producía cierta rutinización de la actividad en el aula que seguía reglas preestablecidas por el docente (Diehl y McFarland, 2012; Mínguez, 2017). La autoridad del docente del siglo XXI no puede fundarse en principios autocráticos definidos por la escuela, sino que se debe legitimar en base un proceso relacional donde cobra una importancia decisiva su capacidad para encarnar la norma institucional 
y para lograr tanto el consenso de los estudiantes como los objetivos que plantea el proyecto educativo del centro.

Por su parte, la ciudadanía ya no se entiende como el conjunto estático de derechos y obligaciones, sino como la facultad de los ciudadanos de intervenir, justamente, en la construcción del conjunto de prerrogativas y responsabilidades que los asisten como miembros de la polis (Jelin, 2003); parte del conjunto de obligaciones y derechos individuales cuyo aprendizaje debe promover y normalizar la escuela. Esto requiere de una intervención docente orientada a actuar a partir de la experiencia cotidiana, promoviendo espacios de reflexión (Dubet, 2010, 2011).

Cárcamo-Vázquez (2015) sostiene que el entorno ideal para la construcción de la ciudadanía, después de la familia, es la escuela porque es el contexto de socialización común de las personas. Además, arguye que la formación ciudadana no solo se debe integrar en el currículo de forma transversal ni mediante la transmisión tradicional de contenidos curriculares en materias específicas sino que debe ser la base de la cultura escolar y de la convivencia cotidiana.

Es necesario que la organización y el funcionamiento de la escuela, las relaciones entre los directivos, profesores, padres y alumnos y los mecanismos de participación de todos los miembros implicados en la vida del centro operen como un lugar de entrenamiento para la participación democrática en la vida adulta (Fernández, 2008, p. 15).

Por supuesto, esto tiene profundas implicaciones respecto a la manera en cómo debe promoverse la ciudadanía en la escuela y con ello en las formas de regulación de los vínculos internos, en la autoridad docente y en cómo se desarrolla la actividad del aula (Míguez, 2017).

Por tanto, es necesario construir una nueva cultura cívica escolar donde las regulaciones de los vínculos internos entre los miembros de la comunidad escolar sean creadas colectivamente e instrumentalizadas mediante el ejercicio democrático de derechos y deberes que involucren al conjunto de sus miembros. Esto implica cuidar que los reglamentos y normativas disciplinarias, que son necesarias para alcanzar los objetivos educativos y para promover la ciudadanía (Mínguez, 2017), no establezcan únicamente obligaciones y sanciones para los estudiantes, sino que promuevan y normalicen el aprendizaje de obligaciones y derechos individuales en la escuela. 
De forma concordante, la autoridad docente para la ciudadanía se entiende como un proceso bidireccional (maestro-alumno) donde no se trata de castigar a quienes incumplan las reglas sino de desarrollar un proceso de negociación democrático continuo. En este sentido, esta investigación nos plantea el reto y la necesitad de construir un espacio de civilidad escolar en el cual los problemas de indisciplina, violencia, disrupción y conflicto se aborden democráticamente, no solo en términos de deberes, como en la escuela tradicional, sino especialmente de derechos (Dubet, 2011).

Entendiendo que la autoridad docente no existe si no es legitimada por sus alumnos, esto es reconocida y aceptada, en este estudio nos proponemos:

1) Identificar los rasgos que caracterizan la autoridad de los maestros, según la opinión del alumnado.

2) Determinar qué tipo de comportamientos generan estos rasgos en los estudiantes.

3) Analizar las posibles relaciones entre la autoridad docente y la formación para la ciudadanía.

\section{Método}

La investigación es analítico-exploratoria. Para la recolección de datos se utilizó el cuestionario denominado Autoridad docente y convivencialidad, diseñado y validado por el Grupo de Investigación de Indisciplina y Violencia en la Educación de la Facultad de Educación de la Universidad de Alicante (España), y que ya fue empleado en otros estudios (Merma-Molina y Gavilán, 2016; Merma-Molina y Gavilán, 2017). Debido a que fue adaptado a los objetivos del estudio, se verificó la pertinencia de los ítems con la participación de 4 investigadores expertos del Área de Teoría e Historia de la Educación.

Con el fin de sintetizar de una manera operativa las valoraciones de los investigadores expertos sobre la adecuación de los indicadores seleccionados, se utilizó el Método De Osterlind (1989) con una escala de tres niveles de congruencia ( -1 si es baja, $0=$ si es media y 1 si es alta). Todos los ítems alcanzaron una congruencia de Osterlind mayor a O,5. Cada ítem tenía cinco posibles valoraciones basadas en la Escala de Likert, que iban desde 1 hasta 5, siendo 1 la puntuación más baja y 5 la más alta.

En atención a las recomendaciones de los investigadores expertos, el ins- 
trumento, que inicialmente tenía 20 ítems, fue modificado añadiéndose en la última versión 10 ítems para indagar las posibles consecuencias que el ejercicio de un determinado estilo de autoridad puede ocasionar en el alumno.

La muestra se realizó de forma aleatoria estratificada por nivel educativo y provincia. Las etapas educativas del muestreo se corresponden con alumnos de Educación Primaria y de Educación Secundaria de la provincia de Alicante (Comunidad Valenciana-España). Los tipos de centros elegidos fueron los públicos, privados y concertados. La muestra estuvo constituida por $\mathrm{n}=738$ estudiantes, 419 alumnos de Educación Primaria (56.78\%) y 319 alumnos de Educación Secundaria (43.22\%).

Las dimensiones que se analizan en la investigación son: 1) Dominio de la asignatura y metodología 2) Conducta ejemplar, 3) Personalidad y 4) Actitudes (Tabla 1).

Tabla 1

Dimensiones analizadas: rasgos de la autoridad docente

\begin{tabular}{|c|c|c|c|c|}
\hline & ACTITUD & $\begin{array}{l}\text { CONDUCTA } \\
\text { EJEMPLAR }\end{array}$ & PERSONALIDAD & $\begin{array}{l}\text { DOMINIO DE LA ASIGNA- } \\
\text { TURA Y METODOLOGÍA }\end{array}$ \\
\hline $\begin{array}{l}\mathrm{C} \\
\mathrm{A}\end{array}$ & $\begin{array}{l}\text { P9: sabe relacionarse } \\
\text { con los demás }\end{array}$ & $\begin{array}{l}\text { P2: su conducta } \\
\text { es conforme lo } \\
\text { que aconseja, da } \\
\text { buen ejemplo }\end{array}$ & $\begin{array}{l}\text { P5: muestra una } \\
\text { gran personalidad, } \\
\text { exige respeto }\end{array}$ & $\begin{array}{l}\text { P1: sabe la asignatura que } \\
\text { explica }\end{array}$ \\
\hline $\begin{array}{l}\mathrm{R} \\
\mathrm{A} \\
\mathrm{C}\end{array}$ & $\begin{array}{l}\text { P12: no es excluyente, } \\
\text { promueve la participa- } \\
\text { ción }\end{array}$ & $\begin{array}{l}\text { P3: respeta a to- } \\
\text { dos los estudian- } \\
\text { tes }\end{array}$ & $\begin{array}{l}\text { P8: es estricto, no } \\
\text { deja pasar una }\end{array}$ & $\begin{array}{l}\text { P4: explica claramente, sin } \\
\text { perderse en cuestiones que } \\
\text { no van con el tema }\end{array}$ \\
\hline $\begin{array}{l}\mathrm{T} \\
\mathrm{E} \\
\mathrm{R} \\
\mathrm{I}\end{array}$ & $\begin{array}{l}\text { P13: tiene interés por- } \\
\text { que todos los alumnos } \\
\text { aprendan }\end{array}$ & $\begin{array}{l}\text { P6: sanciona las } \\
\text { faltas con justicia }\end{array}$ & $\begin{array}{l}\text { P11: es imparcial, } \\
\text { no favorece ni } \\
\text { premia a unos so- } \\
\text { bre los otros }\end{array}$ & $\begin{array}{l}\text { P7: no pide memorismo, } \\
\text { pretende que seamos crea- } \\
\text { tivos }\end{array}$ \\
\hline $\begin{array}{l}S \\
T\end{array}$ & $\begin{array}{l}\text { P16: busca armonía, tie- } \\
\text { ne comunicación aser- } \\
\text { tiva }\end{array}$ & & $\begin{array}{l}\text { P18: es autoexi- } \\
\text { gente, intimidante } \\
\text { y controlador }\end{array}$ & $\begin{array}{l}\text { P10: sus clases son entre- } \\
\text { tenidas, pero no deja de } \\
\text { enseñar }\end{array}$ \\
\hline $\begin{array}{l}\text { C } \\
\text { A } \\
\text { S }\end{array}$ & $\begin{array}{l}\text { P17: a veces es incon- } \\
\text { sistente, impreciso, su } \\
\text { creatividad es limitada }\end{array}$ & & & $\begin{array}{l}\text { P14: cuando hay bajas } \\
\text { calificaciones, explica los } \\
\text { ejercicios }\end{array}$ \\
\hline & $\begin{array}{l}\text { P19: no hace nada, evita } \\
\text { problemas, es apático }\end{array}$ & & & $\begin{array}{l}\text { P15:entrega enseguida los } \\
\text { ejercicios corregidos }\end{array}$ \\
\hline & $\begin{array}{l}\text { P20: busca soluciones a } \\
\text { los conflictos, negocia } \\
\text { con los alumnos }\end{array}$ & & & \\
\hline
\end{tabular}

Fuente: elaboración propia. 
Los datos se recogieron entre los meses de agosto y octubre de 2015 y fueron transcritos y organizados en la plataforma en línea del grupo de investigación. Posteriormente se realizó el análisis e interpretación estadística en el programa SPSS 22.0. En el análisis se hizo hincapié en los estadísticos descriptivos como la media, desviación estándar y en la determinación de las respectivas correlaciones.

\section{Resultados}

Los resultados muestran que lo que más destacan los estudiantes como rasgos de autoridad docente son la Actitud del profesor, dimensión a la que se asignaron 7 de los 20 criterios establecidos en el instrumento.

Tabla 2

Actitudes del profesor

\begin{tabular}{ccccccccc}
\hline MUESTRA & POBLACIÓN & p9 & p12 & p13 & p16 & p17 & p19 & p20 \\
\hline 738 & TODOS & 1.028 & 1.051 & 1.029 & 1.046 & 1.366 & 1.175 & 1.125 \\
\hline
\end{tabular}

Fuente: elaboración propia.

Los hallazgos más prominentes y que más se acercan a la media (1.038), con relación a la actitudes del profesor, son: p13: tiene interés por cada alumno, p9: sabe relacionarse con los alumnos, p16: busca armonía y trato personal con el alumno, y p12: participa en las actividades y no es excluyente. Por otro lado, los valores que más se alejan de la media son p17: es inconsistente, impreciso, su creatividad es limitada, p19: es apático y evita problemas, y p20: busca soluciones a los conflictos.

Con relación al dominio de la asignatura y a la metodología empleada, a los que se les asignó 6 criterios, prevalecen: p15: informa a los alumnos de sus progresos, p10: sus clases son entretenidas, seguido de p7: no pide memorismo y favorece la creatividad, p14: retroalimenta las explicaciones o temas, p4: explica con claridad y p1: domina la asignatura.

Tabla 3

Dominio de la asignatura y metodología

\begin{tabular}{cccccccc}
\hline MUESTRA & POBLACIÓN & P1 & P4 & P7 & P10 & P14 & P15 \\
\hline 738 & TODOS & 0.845 & 0.987 & 1.085 & 1.060 & 1.080 & 1.037 \\
\hline Fuente: elaboración propia & & & & & &
\end{tabular}


Respecto a su personalidad destaca el ítem p8: es estricto. Los valores más alejados son p18: es autoexigente, intimidante y controlador, p11: es imparcial, no favorece ni premia a unos sobre los otros y p5: tiene una gran personalidad.

Tabla 4

Personalidad

\begin{tabular}{cccccc}
\hline MUESTRA & POBLACIÓN & $\mathrm{p} 5$ & $\mathrm{p} 8$ & $\mathrm{p} 11$ & $\mathrm{p} 18$ \\
\hline 738 & TODOS & 0.950 & 1.084 & 1.251 & 1.361 \\
\hline
\end{tabular}

Fuente: elaboración propia.

Finalmente, señalan tres criterios por debajo de la media para la Conducta ejemplar del maestro: p2: da buen ejemplo, p3: respeta a cada estudiante y p6: es justo con las calificaciones y sanciones.

Tabla 5

Conducta ejemplar

\begin{tabular}{ccccc}
\hline MUESTRA & POBLACIÓN & $\mathrm{p} 2$ & $\mathrm{p} 3$ & $\mathrm{p} 6$ \\
\hline 738 & TODOS & 0.906 & 0.948 & 0.940 \\
\hline
\end{tabular}

Fuente: elaboración propia.

En lo concerniente a las correlaciones, que han permitido medir las relaciones positivas entre las cualidades asignadas a los maestros y los comportamientos del alumno, destaca el ítem p29: los estudiantes quieren al profesor y están contentos, excepto con el p8: "no es blando, es estricto, su relación con los alumnos es inconsistente y su creatividad es limitada, es intimidador y controlador, y es apático e ignora a los estudiantes". Otros comportamientos positivos como consecuencia de la autoridad de los maestros descritos por los propios alumnos se refieren a los ítems p23, p21 y p22: los estudiantes cumplen las normas de clase, estudian la asignatura, y rinden y sacan buenas notas, excepto con la característica "es estricto, no deja pasar una".

Por su parte, las correlaciones negativas se refieren a los criterios vinculados con p25: cuando el maestro está en el aula, hay desorden y gritos; p26: los alumnos son apáticos, pasan de todo; p27: los estudiantes no atienden las explicaciones ni las actividades y p28: hay riñas en el aula entre alumnos. 
Análisis de las valoraciones del alumnado para repensar la autoridad docente y la formación para la ciudadanía

Gladys Merma-Molina y Diego Gavilán Martín

Tabla 6

Correlaciones entre las características del profesor y las actitudes de los estudiantes

ACTITUDES DE LOS ALUMNOS

\begin{tabular}{|c|c|c|c|c|c|c|c|c|c|c|}
\hline & & 1 & 2 & 3 & 24 & 25 & 26 & P27 & 28 & 9 \\
\hline & P1 & .113 & 111 & 0.208 & 118 & -0.230 & & .097 & -0 & 40 \\
\hline & P2 & 6 & 71 & 41 & 29 & 76 & 06 & 79 & 8 & 0.463 \\
\hline & $\mathbf{P}$ & ) & 4 & 5 & 93 & 53 & 54 & 76 & -0 & 0411 \\
\hline & $\mathbf{P}$ & 0.189 & 0.126 & & 9 & 63 & & 8 & & 0.345 \\
\hline & P5 & 5 & 00 & 4 & 1 & 11 & 3 & -0.158 & -0 . & 2 \\
\hline & P6 & 00 & 0.007 & 0.088 & 0.147 & 68 & -0. & -0 . & -0. & 0.299 \\
\hline & $\mathbf{P}$ & 3 & 7 & 43 & 2 & 51 & -0 & -0. & -0. & 6 \\
\hline & $\mathbf{P}$ & 9 & 9 & 8 & -1 & 2 & 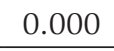 & 4 & -( & \\
\hline & P9 & 0 & 83 & 17 & 9 & 81 & -0 & -0. & -0 & 0 \\
\hline & P10 & 0.248 & 0.166 & 0.191 & 0.114 & -0.376 & -0.227 & -0.210 & -0.133 & 0.427 \\
\hline & P11 & 0.177 & 0.091 & 0.273 & 0.083 & -0.261 & -0.196 & -0.205 & -0.109 & 0.347 \\
\hline & P12 & & 0030 & & & -0.189 & & -0.123 & -0.047 & 0391 \\
\hline & P13 & 0 & 0 & & & & 4 & -0 & 34 & 3 \\
\hline & P14 & 0.287 & 0.120 & 0.191 & 0.0 & -0.269 & -0.210 & -0.124 & 81 & 0.479 \\
\hline & P15 & 0.185 & 0.177 & 0.140 & 0.031 & -0.216 & -0.209 & -0.130 & -0.078 & 0.268 \\
\hline & P16 & 0.263 & 0.056 & 0194 & 0.115 & -0.353 & -0.300 & -0.251 & -0.1 & 0 \\
\hline & P17 & .052 & 0.029 & -0.034 & 0.02 & 0.270 & 0.2 & 0.20 & 53 & -0. \\
\hline & P18 & -0.081 & -0.054 & -0.070 & -0.047 & 0.270 & 0.297 & 0.263 & 0.247 & -0.246 \\
\hline & P19 & -0.160 & 0.022 & -0.112 & -0.116 & 59 & 27 & 0.303 & 0.245 & -0.336 \\
\hline & P20 & 67 & 78 & 13 & 75 & 79 & 3 & 6 & 4 & 0 \\
\hline
\end{tabular}

Fuente: elaboración propia.

\section{Discusión y conclusiones}

Los objetivos de este estudio fueron determinar los rasgos que caracterizan la autoridad de los profesores, según la opinión de los alumnos, identificar qué tipo de comportamientos generan estos rasgos en los estudiantes, y analizar las posibles relaciones entre la autoridad docente y ciudadanía.

Los hallazgos del estudio permiten concluir que la dimensión más valorada por los estudiantes como rasgos de la autoridad del docente 
son sus actitudes (p9, p12, p13, p16, p17, p19 y p20), concretamente sus habilidades para relacionarse con los alumnos, su carácter inclusivo, su capacidad para solucionar situaciones problemáticas, y su interés por buscar armonía y trato personal con los estudiantes. Si el docente no le da la debida importancia a las opiniones de sus alumnos, será muy difícil captar su atención o lograr que participen activamente y que expresen abierta y críticamente sus ideas. Para promover los procesos de "ciudadanización en la escuela" es crucial que los maestros desarrollen habilidades de comunicación y de resolución de conflictos (CarlssonPaige, y Lantieri, 2004).

La segunda dimensión destacada por el alumnado son las metodologías activas que emplea el docente, su buena formación, su interés porque aprendan y su dominio de la asignatura (p1, p4, p7, p10, p14 y p15). Estos resultados concuerdan con el estudio de Harjunen (2011) que concluye que los estudiantes de escuelas secundarias finlandesas valoran, después la dimensión relacional, el apoyo y la motivación de los profesores hacia el aprendizaje, a diferencia del estudio de Zamora-Poblete, Meza-Pardo y Cox-Vial (2015), realizado con estudiantes chilenos de Educación Secundaria, donde se muestra que la autoridad docente no está asociada directamente con el profesor sino con el tipo de asignatura que enseña y con la relevancia de la materia para los estudiantes.

Respecto a la tercera dimensión, personalidad del docente, los participantes destacan que es estricto (p8), autoexigente (p18) e imparcial (p11). Finalmente, la dimensión "conducta ejemplar del maestro" tiene una escasa valoración (p2: su conducta es conforme a lo que aconseja, p6: sanciona faltas con justicia y p3: respeta a cada uno de los estudiantes). Estos hallazgos concuerdan con el Informe del Defensor del Menor (2006) donde se concluye que el alumnado percibe que más de la mitad de los maestros muestran favoritismos con ciertos compañeros y que aplican las normas de forma desigual; según este informe, el 16\% de adolescentes consideran que sus profesores "les tienen manía" y un 7\% señalan que se sienten ridiculizados por el profesor.

Si el docente representa la disciplina, el orden, la dedicación y el verdadero interés por las personas, es necesario que haya una coherencia entre lo que "dice, hace y es". Esto supone trasladar a la vida personal las mismas actitudes que exige en clase; por ejemplo, es fácil pedir que los alumnos cumplan las actividades, que entreguen las tareas académicas en los plazos establecidos y que sean ordenados, pero esto implica 
que también el docente revise, corrija, haga observaciones y entregue los resultados con la misma puntualidad solicitada. Las actitudes de los alumnos muchas veces son el reflejo de la personalidad del profesor, por lo que educar con el ejemplo es fundamental.

A partir del análisis de las correlaciones, se concluye que los estudiantes demandan una autoridad legítima, relacional e interpersonal del maestro, que solo puede afirmarse a partir de la práctica de relaciones democráticas cercanas entre ambos actores educativos, basadas en el respeto y de confianza mutua.

Los estudiantes valoran como rasgos de la autoridad docente especialmente el interés que muestran sus profesores por ellos -individual y no de grupo- y su cercanía y su capacidad para establecer una comunicación asertiva (p14, p9, p20, p16 y p29); a su vez, estas actitudes de los maestros provocan que los alumnos estén a gusto con él (p23), que cumplan las normas de clase (p23) y que estudien la asignatura (p21). Por el contrario, hay problemas de convivencia en el aula, especialmente de indisciplina - de no aceptación y de incumplimiento de las normas- (p25, p28) y desinterés y apatía por las actividades realizadas por el profesor (p27) cuando este es inconsistente, impreciso y tiene poca creatividad (p17), es intimidante y controlador (p18), y no hace nada, evita problemas y es apático (p19). Esto significa que las estrategias frecuentemente utilizadas por los docentes cuando se producen situaciones de indisciplina en el aula, como son el abuso de poder y el excesivo control, impiden el desarrollo activo y libre del alumno, y limita o anula la responsabilidad del discente en el proceso educativo (Lago y RuizRoso, 2017). En cambio, si el docente tiene en cuenta las características, necesidades, preferencias e intereses de cada estudiante -como un ser individual-, su autoridad se hace legítima (Roberson, 2014).

Los hallazgos del estudio también apoyan la idea de que la mejora del buen comportamiento, del interés por el estudio y de la adquisición de hábitos positivos en los alumnos es directamente proporcional con la autoridad del maestro; esto significa que las actitudes positivas o negativas del profesor son predictores positivos o negativos de la conducta de los alumnos. Los estudiantes interpretan la autoridad como una relación que se sostiene en el reconocimiento recíproco entre las partes. La reciprocidad se trasluce en el deseo de recibir algo a cambio de la aceptación de la autoridad: respeto, atención o reconocimiento.

Por tanto, la autoridad del maestro no es legítima porque encarne -a 
Análisis de las valoraciones del alumnado para repensar la autoridad docente y la formación para la ciudadanía

Gladys Merma-Molina y Diego Gavilán Martín

priori- la autoridad impuesta por la escuela, sino porque es capaz de ser querido y respetado por sus alumnos y porque es eficaz en su trabajo (Dubet, 2011). La autoridad surge a partir de la relación social entre el maestro y el alumno, que se manifiesta en la "obediencia aceptada". Lograr que los estudiantes "obedezcan" a sus profesores no es algo trivial, por el contrario es fundamental para el clima escolar y para el rendimiento académico.

El desarrollo de habilidades y aptitudes para el ejercicio de la autoridad docente y de la ciudadanía en aula se adquiere en la formación inicial y se afianza en el desarrollo profesional del docente. Consecuentemente, las acciones pedagógicas cotidianas del aula deben centrarse en las oportunidades para poner en práctica estas habilidades en todos los entornos de aprendizaje, fundamentalmente en la universidad y la escuela.

\section{Referencias}

Álvarez, M. Á., Castro, P., González-González, C., Martino, E. y Campo, Á. (2016). Conductas disruptivas desde la óptica del docente: validación de una escala. Anales de Psicología, 32(3), 855-862.

Arendt, H. (1996). Entre el pasado y el futuro. Barcelona: Península.

Barba, J. J. (2009). Redefiniendo la autoridad en el aula. Posibilidades para una educación democrática. Retos. Nuevas Tendencias en Educación Física, Deporte y Recreación, 15, 41-44.

Calvo, A. (2011). Conductas disruptivas y gestión eficaz del aula. Departamento de Psicología Evolutiva y de la Educación, Universidad de Murcia, España. Recuperado de http:// www.ceptorrelavega.educantabria.es

Cárcamo-Vásquez, H. (2015). Ciudadanía y formación ciudadana: ¿Qué ocurre en el aula donde se forma el futuro profesorado de educación primaria de una universidad pública de Madrid? Revista Electrónica Educare. Educare Electronic Journal, 19(3), 1-14.

Carlsson-Paige, N. \& Lantieri, L. (2004). A changing vision of education [Una visión cambiante de la educación]. En N. Noddings (Ed.), Educating ctizens for global awareness [Educando ciudadanos para la conciencia global] (pp. 107-121). New York: Teachers College Press.

Defensor del Menor. (2006). Convivencia, conflictos y educación en centros escolares de la comunidad de Madrid. Madrid: Publicaciones del Defensor del Menor de la Comunidad de Madrid.

Díaz, C. (1998). Diez palabras clave para educar en valores. Salamanca: Kadmos.

Díaz-Aguado, M. J. (2013). Convivencia escolar y prevención de la violencia. España: Ministerio de Educación, Cultura y Deporte. 
Análisis de las valoraciones del alumnado para repensar la autoridad docente y la formación para la ciudadanía

Gladys Merma-Molina y Diego Gavilán Martín

Díaz-Aguado, M. J., Martínez, R. y Martín, J. (2010). Estudio estatal sobre la convivencia escolar en la Educación Secundaria Obligatoria. Madrid: MEC.

Diehl, D. \& McFarland, D. (2012). Classroom ordering and the situational imperatives of routine and ritual. Sociology of Education, 85(4), 326-249.

Dubet, F. (2006). El declive de la institución. Barcelona: Gedisa.

Dubet, F. (2007). El declive y las mutaciones de la institución. Revista de Antropología Social, 16, 39-66.

Dubet, F. (2010). Sociología de la experiencia. Madrid: Centro de Investigaciones Sociológicas.

Dubet, F. (2011). Mutações cruzadas: a cidadania e a escola. Revista Brasileira de Educação. 16(47), 290-305.

Espot, R. M. (2006): La autoridad del profesor. Qué es la autoridad y cómo se adquiere. Madrid: Editorial Praxis.

Fernández, M. (2008). Escuela y ciudadanía en la era global. En G. Frigerio y G. Diker (Comps.), Educar: posiciones acerca de lo común (pp. 15-31). Buenos Aires: Del Estante Editorial.

Gallego, L., Acosta, J., Villalobos, Y., López, A. y Giraldo, A. (2016). Violencia del docente en el aula de clase. Revista de Investigaciones. UCM, 16(2), 116-125.

Harjunen, E. (2009). How do teachers view their own pedagogical authority? Teachers and Teaching: Theory and Practice, 15(1), 87-107.

Harjunen, E. (2011). Students' consent to a teacher's pedagogical authority. Scandinavian Journal of Educational Research, 55(4), 403-424.

Ivanenko, N. (2017). Education for democratic citizenship: teaching virtues and values. Наукові записки [Кіровоградського державного педагогічного університету імені Володимира Винниченка]. Серія: Педагогічні науки, 152, 110-113.

Jelin, E. (2003). Citizenship and alterity. Tensions and dilemmas. Latin American Perspectives, 30(2), 309-325.

Kojève, A. (2006). La noción de autoridad. Madrid: Nueva Visión.

Ladson-Billings, G. (2004). Differing concepts of citizenship: Schools and communities as sites of civic development [Diferentes conceptos de ciudadanía: escuelas y comunidades como espacios de desarrollo cívico]. En N. Noddings (Ed.), Educating citizens for global awareness [Educando ciudadanos para la conciencia global] (pp. 69-80). New York: Teachers College Press.

Lago, J. C. y Ruiz-Roso, L. (2017). Autoridad y control en el aula: de la disciplina escolar a la disciplina judicial. Tarbiya, Revista de Investigación e Innovación Educativa, 25, 49-93.

Maguire, M., Ball, S. \& Braun, A. (2010). Behaviour classroom management and student «control»: enacting policy in the English secondary school. International Studies in Sociology of Education, 20(2), 153-170.

Maphosa, C. \& Mammen, K. J. (2011). How chaotic and unmanageable classrooms have become: Insights into prevalent forms or learner indiscipline in South African Schools. Anthropologist, 13(3), 185-193.

Martuccelli, D. (2016). Condición adolescente y ciudadanía escolar. Educação \& Realidade, 41(1), 155-174.

McGarr, O., O'Grady, E. \& Guilfoyle, L. (2016). Exploring the theory-practice gap in ini- 
Análisis de las valoraciones del alumnado para repensar la autoridad docente y la formación para la ciudadanía Gladys Merma-Molina y Diego Gavilán Martín

tial teacher education: moving beyond questions of relevance to issues of power and authority. Journal of Education for Teaching, 43(1), 48-60.

McMahon, S. D., Martínez, A., Espelage, D., Rose, C., Reddy, L. A., Lane, K., ... y Brown, V. (2014). Violence directed against teachers: Results from a national survey. Psychology in the Schools, 51(7), 753-766.

Merma-Molina, G. \& Gavilán, D. (2016). Perceptions of students on the authority of the teacher in the classroom. Leading education: The distinct contributions of educational research and researchers. Dublín: European Educational Research Association (ECER).

Merma-Molina, G. y Gavilán, D. (2017). Caracterización de la autoridad del docente universitario y sus repercusiones en el alumnado de Magisterio: Caso de estudio. En R. Roig (2017), Investigación en docencia universitaria: Diseñando el futuro a partir de la innovación educativa (pp. 321-328). Barcelona: Octaedro.

Míguez, D. P. (2017). Las complejidades de la convivencia ciudadana en la Escuela Media Argentina. Un estudio de caso en la provincia de Buenos Aires. Polyphōnía. Revista de Educación Inclusiva, 1(1), 23-45.

Osterlind, S. J. (1989). Constructing test ítems. Boston: Kluwer.

Palomares, A. (2008). Educación para la ciudadanía y la convivencia. Contextos Educativos. Revista de Educación, 11, 71-94.

Pierella, M. P. (2015). La autoridad de los profesores universitarios: un estudio centrado en relatos de estudiantes de letras. Educação e Pesquisa, 41(2), 427-442.

Roberson, R. R. (2014). Understanding the development of legitimate teacher authority through the teacher-student relationship: A qualitative study (Doctoral thesis). University of Oklahoma, Oklahoma.

Ruiz, A. P. (2013). Educación para la ciudadanía y la convivencia. Contextos Educativos. Revista de Educación, 11, 71-94.

Simón, C., Gómez, P. y Alonso, J. (2013). Prevención de la disrupción en el aula: papel del clima motivacional de clase y de las estrategias de afrontamiento. Cultura y Educación, 25(1), 49-64. doi:10.1174/113564013806309037

Tahull, J. y Montero, Y. (2013). Reflexionando sobre el concepto de autoridad. RASE: Revista de la Asociación de Sociología de la Educación, 6(3), 459-477.

Tassin, É. (2011). Educar al ciudadano: ¿Qué éxito se puede esperar de un oficio imposible? En Varios autores (Ed.), Memorias del seminario internacional ciudadanía y convivencia: un espacio de reflexión desde la educación y la pedagogía (pp. 10-13). Bogotá: Alcaldía Mayor de Bogotá Educación.

Torrubia, E., Guzón, J. L. y Alfonso, J. M. (2017). Padres y escuelas que hacen crecer en el siglo XXI. Alteridad. Revista de Educación, 12(2), 215-223.

Urbina, C., Simón, C. y Echeita, G. (2011). Concepciones de los profesores acerca delas conductas disruptivas: análisis a partir de un marco inclusivo. Infancia y Aprendizaje: Journal for the Study of Education and Development, 34(2), 205-217. doi:10.1174/021037011795377584.

Zamora, G. y Zerón, A. (2010). Caracterización y sentido actual de la autoridad pedagógica en escuelas chilenas de sectores de pobreza. Revista Española de Pedagogía, 68(245), 99-116. 
Análisis de las valoraciones del alumnado para repensar la autoridad docente y la formación para la ciudadanía

Gladys Merma-Molina y Diego Gavilán Martín

Zamora, G. y Zerón, A. (2010). Caracterización y sentido actual de la autoridad pedagógica en escuelas chilenas de sectores de pobreza. Revista Española de Pedagogía, 68(245), 99-116. 